\title{
Supporting information: Spontaneous light emission assisted by Mie resonances in diamond nanoparticles
}

\author{
Dmitry V. Obydennov, ${ }^{1}$ Daniil A. Shilkin, ${ }^{1}$ Ekaterina I. Elyas, ${ }^{1}$ \\ Vitaly V. Yaroshenko, ${ }^{2}$ Oleg S. Kudryavtsev ${ }^{3}$ Dmitry A. Zuev, ${ }^{2}$ Evgeny V. Lyubin, ${ }^{1}$ \\ Evgeny A. Ekimov, ${ }^{4,5}$ Igor I. Vlasov, ${ }^{3}$ and Andrey A. Fedyanin ${ }^{1, *}$ \\ ${ }^{1}$ Faculty of Physics, Lomonosov Moscow State University, Moscow 119991, Russia \\ ${ }^{2}$ School of Physics and Engineering, ITMO University, St. Petersburg 191002, Russia \\ ${ }^{3}$ Prokhorov General Physics Institute, Russian Academy of Sciences, Moscow 119991, Russia \\ ${ }^{4}$ Institute for High Pressure Physics, Russian Academy of Sciences, Troitsk 142190, Russia \\ ${ }^{5}$ Lebedev Physical Institute, Russian Academy of Sciences, Moscow 117924, Russia
}

\section{Section I. Fabrication of diamond particles}

Mixture of Adamantane $\mathrm{C}_{10} \mathrm{H}_{16} \quad(\geq 99 \%$, Sigma-Aldrich) and Tetraphenylsilane $\mathrm{C}_{24} \mathrm{H}_{20} \mathrm{Si}$ (96\%, Alfa Aesar) at about 80:1 mass ratio was used for the synthesis of diamonds doped with silicon. The mixture was prepared by mechanical stirring using a mortar and pestle made of Plexiglas. Concentrations of dopant elements relative to carbon: $\mathrm{S} /(\mathrm{Si}+\mathrm{C})$ in atomic percentages was calculated as $0.05 \%$.

The synthesis of the sample was carried out in titanium capsules at a pressure of 8-9 GPa and a temperature of about $1900 \mathrm{~K}$; the exposure time at constant $P$ and $T$ did not exceed $10 \mathrm{~s}$. The toroidtype chamber ("toroid-15") was calibrated by pressure at room temperature using phase transitions in $\mathrm{Bi}(7.7 \mathrm{GPa})$ and $\mathrm{Sn}(9.4 \mathrm{GPa})[1]$. The temperature in the experiment was monitored using a chromel-alumel thermocouple up to $1600 \mathrm{~K}$ and a tungsten-rhenium thermocouple (WRe 5/20) to a temperature of $1900 \mathrm{~K}$ with junctions of the thermocouples mounted at the outer wall of the heater in the middle of its height. The mass of material loaded into the capsule was about $60 \mathrm{mg}$; material was loaded in the form of a compressed tablet with a diameter of $5 \mathrm{~mm}$ and a height of $2.6 \mathrm{~mm}$. The design of the high-pressure cell and the synthesis details are described in Ref. 1.

The synthesized samples were purified from nondiamond phases in boiling perchloric acid $\mathrm{HClO}_{4}$ for 16 hours. Then the acid was washed out with distilled water. After the fabrication procedures were performed, the diamond particles were dispersed on a cover glass from water suspension.

\footnotetext{
${ }^{*}$ Electronic address: fedyanin@nanolab.phys.msu.ru
}

\section{Section II. Calculations}

Three-dimensional finite-difference timedomain numerical simulations were performed using Lumerical FDTD Solutions software. The simulation geometry consisted of a diamond particle located on an amorphous silica substrate. We used this method to model scattering spectra, internal pump field enhancement, and Purcell enhancement taking into account the presence of the substrate and the experimental conditions of excitation and collection.

When simulating scattering spectra, we used a plane wave as a source; it illuminated the particle at normal incidence from the side of the air. The scattering spectra were found as optical power in the far field, integrated over the angles corresponding to the numerical aperture range from 0.17 to 1.2 (the upper value is the numerical aperture of the collecting objective, and the lower value is determined by the central stop diameter used in the experiment).

In the simulations of the dipole radiation enhancement, an electric dipole source was placed within the particle volume. The Purcell factor was found as the power radiated by the dipole in the studied system divided by the power radiated by the same dipole in bulk diamond. Figure S1 demonstrates the calculated Purcell factor at a wavelength of $738 \mathrm{~nm}$ for three different dipole positions within the hosting spherical particle. Depending on the position and orientation of the dipole, the emission is coupled to various modes of the particle. When the source is located at the particle center, the emission is primarily coupled to the electric dipole mode. When it is displaced from the center, the magnetic dipole and quadrupole modes begin to work. As a result, 

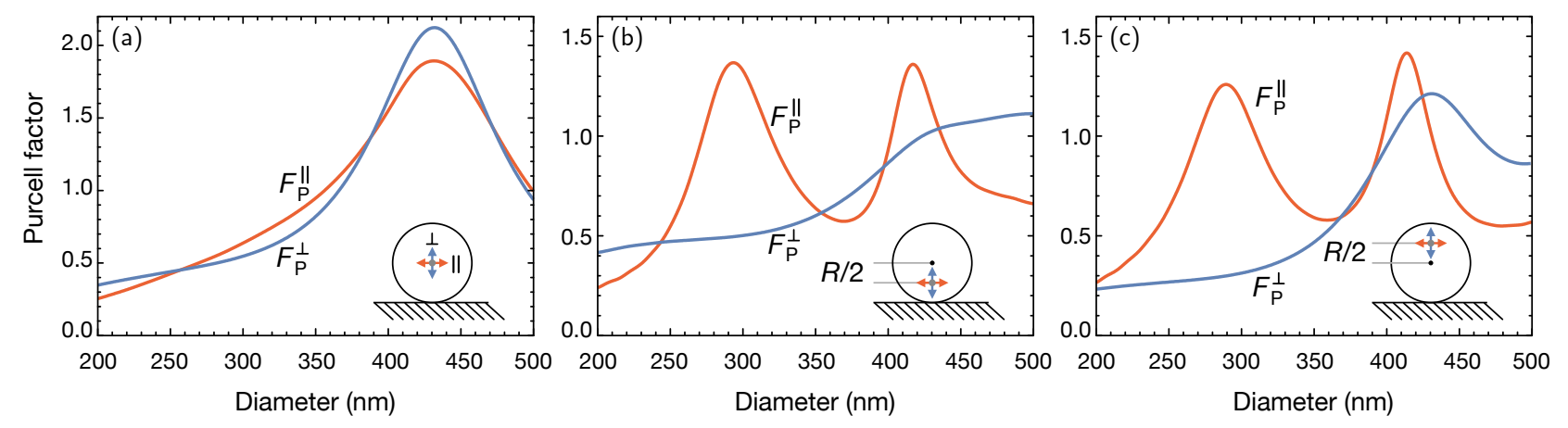

Figure S1: Numerically calculated Purcell factor at $738 \mathrm{~nm}$ as a function of the hosting diamond size. (a) The emitter is placed in the center of the particle. (b,c) The emitter is displaced by half a radius (b) downward and (c) upward from the particle center.

the maximum Purcell factor is achieved at different particle diameters depending on the position and orientation of the dipole.

When describing the experimentally observed Raman enhancement and lifetime modifications, one needs to take into account the emission direction. To calculate the emission power directed to the collection optics, we used plane monitors recording the radiated fields above and behind the particle, as shown in Figure S2. Based on the recorded data, electromagnetic fields in the farfield zone were found. Then, the far-field intensity was integrated over the experimental collection angles and normalized to the source power. The found measure was used as a weighting factor $I_{j}$ to obtain the averaged Purcell factor shown in Figure 2c of the main text; in that case, we modeled a dipole source emitting at the wavelength of $738 \mathrm{~nm}$, the fields were recorded in the upper monitor and integrated over a numerical aperture of 0.7 . The dependence shown in Figure 3c in the main text was obtained using a dipole source emitting at the wavelength of $573 \mathrm{~nm}$, the bottom monitor data, and far-field integration over a numerical aperture of 1.2 .

In the simulations of the pump field enhancement, a Gaussian beam source was used. The beam was directed from the side of the substrate and focused in the particle center with a numerical aperture of 0.42 that corresponds to the experimental illumination conditions. For each particle size, electric fields were recorded and averaged over the particle volume. In this way, the dependence shown in Figure 3b in the main text was obtained.

In order to interpret the results of the numerical calculations, we performed analytical calculations

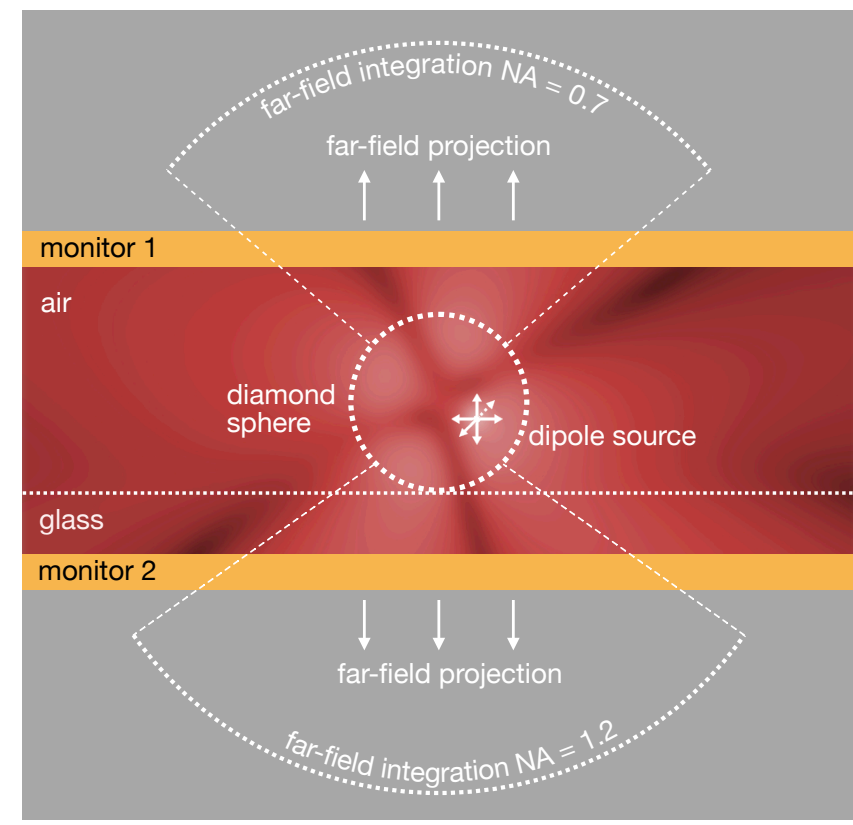

Figure S2: Geometry of numerical simulations of the dipole emission.

for a diamond sphere in a vacuum using Mie theory [2]. The scattering efficiency $Q$ for a sphere of radius $a$ was calculated as follows:

$$
Q=\frac{2}{x^{2}} \sum_{n=1}^{\infty}(2 n+1)\left(\left|a_{n}\right|^{2}+\left|b_{n}\right|^{2}\right),
$$

where $x=k a=2 \pi a / \lambda$, and $\lambda$ is the wavelength of light in vacuum; coefficients $a_{n}$ and $b_{n}$ are defined in the following way:

$$
\begin{aligned}
& a_{n}=\frac{m \psi_{n}(m x) \psi_{n}^{\prime}(x)-\psi_{n}(x) \psi_{n}^{\prime}(m x)}{m \psi_{n}(m x) \xi_{n}^{\prime}(x)-\xi_{n}(x) \psi_{n}^{\prime}(m x)}, \\
& b_{n}=\frac{\psi_{n}(m x) \psi_{n}^{\prime}(x)-m \psi_{n}(x) \psi_{n}^{\prime}(m x)}{\psi_{n}(m x) \xi_{n}^{\prime}(x)-m \xi_{n}(x) \psi_{n}^{\prime}(m x)},
\end{aligned}
$$



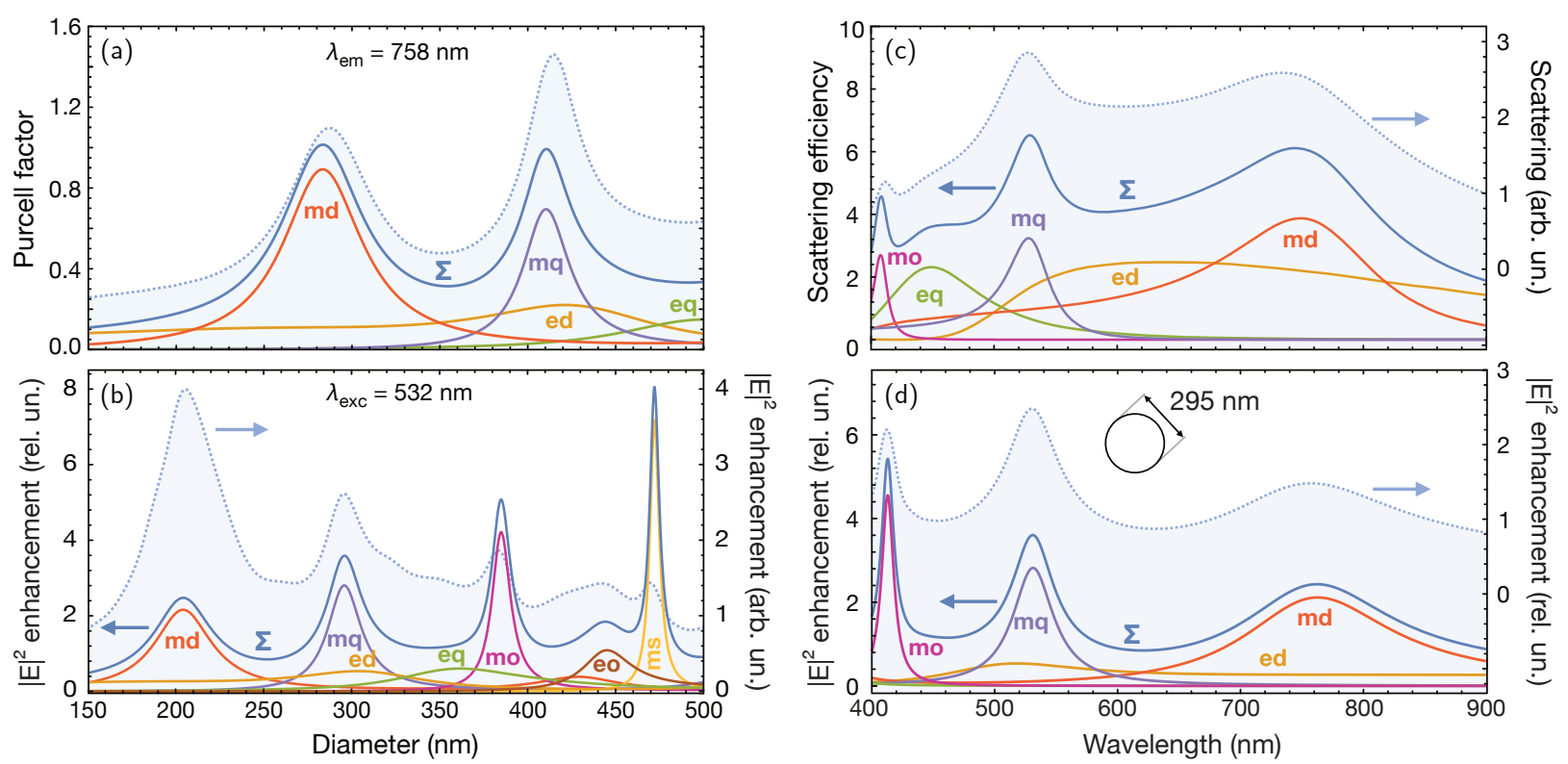

Figure S3: Analytical (solid curves) and numerical (shaded areas) calculations of Mie resonances in diamond spheres. The analytical calculations are performed for a sphere in a vacuum, while the numerical ones take into account the substrate effects. Multipole expansion of the analytical dependences is shown by colored curves and indicated by the following notations: 'md', magnetic dipole; 'mq', magnetic quadrupole; 'mo', magnetic octupole; 'ms', magnetic sedecipole; 'ed', electric dipole; 'eq', electric quadrupole; 'eo', electric octupole; the overall results are indicated by blue solid curves and the symbol $\boldsymbol{\Sigma}$. (a) Purcell factor at a wavelength of $738 \mathrm{~nm}$. (b) Pump field enhancement at a wavelength of $532 \mathrm{~nm}$. Analytical calculations are obtained for plane wave illumination, and the numerical ones are for a Gaussian beam. (c) Light-scattering spectrum of a 295-nm spherical diamond under plane wave illumination. (d) Pump field enhancement spectrum for a 295-nm spherical diamond under plane wave illumination.

where $m$ is the refractive index of the sphere, and $\psi_{n}(\rho)=\rho j_{n}(\rho), \xi_{n}(\rho)=\rho h_{n}^{(1)}(\rho)$ are RiccatiBessel functions expressed in terms of the spherical Bessel functions $j_{n}(\rho)$ and Hankel functions $h_{n}^{(1)}(\rho)$. The electric field power enhancement averaged over the sphere volume $\zeta$ can be expressed as follows:

$$
\begin{aligned}
\zeta & =\left\langle\frac{|E|^{2}}{\left|E_{0}\right|^{2}}\right\rangle_{V}= \\
& =\frac{3}{2(m x)^{3}} \sum_{n=1}^{\infty}(2 n+1)\left(K_{n}\left|c_{n}\right|^{2}+J_{n}\left|d_{n}\right|^{2}\right),
\end{aligned}
$$

where coefficients $c_{n}$ and $d_{n}$ are

$$
\begin{aligned}
c_{n} & =\frac{m \psi_{n}(x) \xi_{n}^{\prime}(x)-m \xi_{n}(x) \psi_{n}^{\prime}(x)}{\psi_{n}(m x) \xi_{n}^{\prime}(x)-m \xi_{n}(x) \psi_{n}^{\prime}(m x)}, \\
d_{n} & =\frac{m \psi_{n}(x) \xi_{n}^{\prime}(x)-m \xi_{n}(x) \psi_{n}^{\prime}(x)}{m \psi_{n}(m x) \xi_{n}^{\prime}(x)-\xi_{n}(x) \psi_{n}^{\prime}(m x)},
\end{aligned}
$$

and $K_{n}, J_{n}$ are

$$
\begin{aligned}
K_{n} & =\left(m x^{3} / 2\right)\left[j_{n}^{2}(m x)-j_{n+1}(m x) j_{n-1}(m x)\right], \\
J_{n} & =K_{n-1}-n m x j_{n}^{2}(m x) .
\end{aligned}
$$

Finally, for analytical calculations of Purcell factor, we used the results reported in Ref. 3 .

Figure S3 demonstrates the obtained analytical dependences in comparison with the numerical ones. One can see that the presence of the substrate only slightly modifies the studied dependences, and the observed peaks can be associated with the excitation of specific Mie modes. Figure S3b also demonstrates the importance of taking into account the beam profile when calculating the pump field enhancement. Because of the Gaussian beam used in the model, the excitation efficiency of higher-order resonances is significantly lower. Analytical calculations performed for the plane-wave excitation, in contrast, show a stronger field enhancement for higher-order resonances. Figures S3c and S3d show the scattering and field enhancement spectra, respectively, calculated for the same diamond sphere with a diameter of $295 \mathrm{~nm}$. Note that the field enhancement maxima do not exactly coincide with the scattering peaks, even for a spherical diamond without any substrate. 


\section{Section III. Scattering spectroscopy and particle size analysis}

During scattering spectroscopy of the sample diamonds, two spectra were recorded for each particle: the intensity of dark-field scattering at the particle $I_{\text {particle }}$ and the intensity from an empty substrate near the particle $I_{\text {substrate }}$. The scattering spectra were then obtained as follows:

$$
f_{\exp }=\frac{I_{\text {particle }}-I_{\text {substrate }}}{I_{\text {lamp }}-I_{\text {noise }}},
$$

where $I_{\text {lamp }}$ is the reference lamp spectrum and $I_{\text {noise }}$ is dark counts. We fitted the measured spectra by numerical ones to extract the effective size of the studied particles. For each measured spectrum, we have found the following function:

$$
\chi^{2}(d, D)=\int \frac{\left(f_{\exp }(\lambda)-D f_{\text {num }}(\lambda, d)\right)^{2}}{\sigma(\lambda)} d \lambda,
$$

where $\lambda$ is the wavelength, $f_{\exp }(\lambda)$ is the measured scattering spectrum, $f_{\text {num }}(\lambda, d)$ is the scattering spectrum calculated for a spherical particle, $d$ is the particle diameter, $D$ is the scaling factor, and $\sigma(\lambda)$ is the experimental data error squared. We used $d$ and $D$ as adjustable parameters to minimize $\chi^{2}$, and the resulting $d$ value was designated as the effective particle size. The error bars shown in the experimental graphs correspond to a two-fold increase in $\chi^{2}$ compared to the minimum value.

After all the optical measurements were performed, we also studied the sample using scanning electron microscopy. Figure S4a demonstrates the scattering spectra for a number of the studied particles along with their scanning electron micrographs. Numerically calculated spectra best fitting the experimental ones are shown in Figure S4b; the determined effective size values are indicated in the bottom right corners. One can see that in the scanning electron micrographs, particles with smaller effective sizes appeared to be smaller.

To validate this observation quantitatively, we have analyzed scanning electron micrographs in the following way. For each image, we found the diameter of the largest circle that can be inscribed in the particle contour using Fiji software [4], Max Inscribed Circle plugin. Figure S5 shows the results of this analysis. The obtained values are plotted along the horizontal axis, while the effective size values extracted from the scattering spectra are

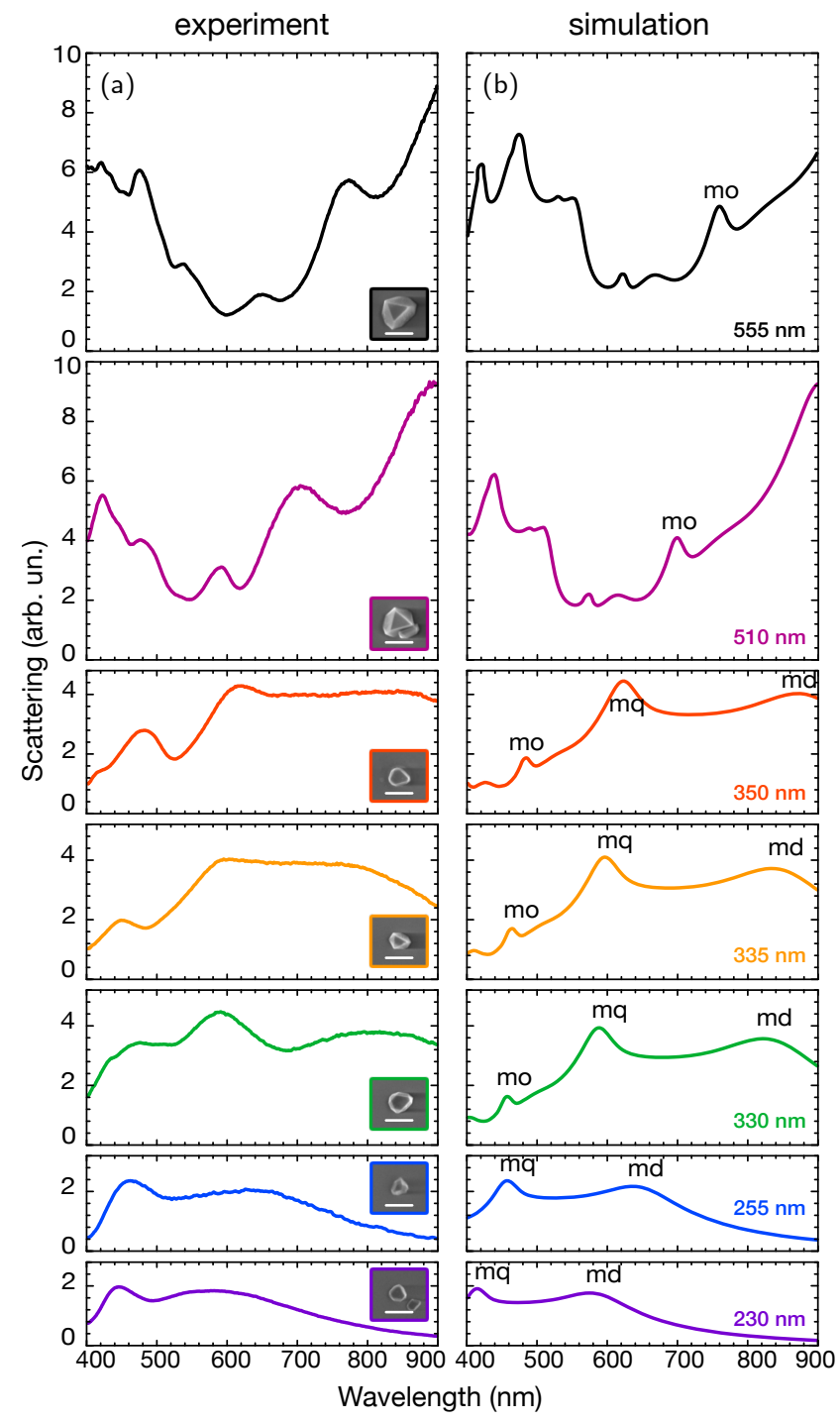

Figure S4: Scattering spectroscopy and scanning electron microscopy of individual diamonds. (a) Experimental scattering spectra. Scanning electron micrographs of the corresponding particles are shown in the insets. The scale bar is $500 \mathrm{~nm}$. (b) Calculated scattering spectra of diamond spheres on a glass substrate. The diameters used are shown on the graphs and correspond to the effective diameters of the particles shown in panel (a). Notations 'md', 'mq', and 'mo' indicate the positions of the magnetic dipole, quadrupole, and octupole resonances, respectively.

measured along the vertical axis. The inset shows an example of a diamond particle image and the corresponding largest circle inscribed. The resulting dependence is well approximated by a linear function; the obtained slope is $1.05 \pm 0.16$, and the intercept is $-40 \pm 50 \mathrm{~nm}$. Thus, we conclude that scattering spectroscopy can be used as an optical method for determining the size of the studied di- 


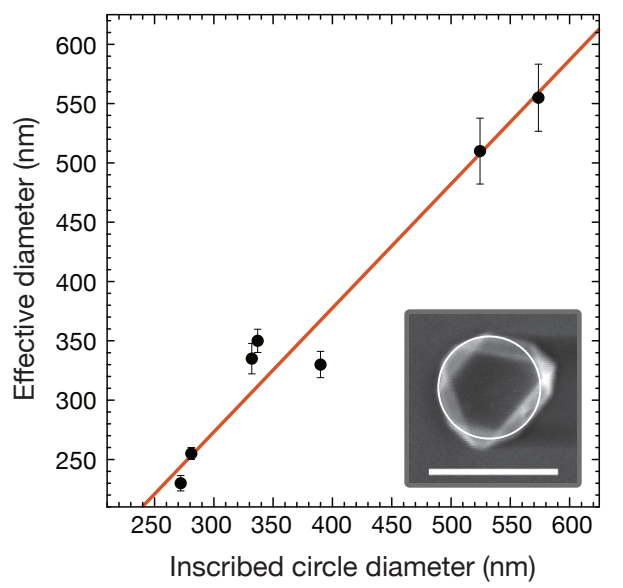

Figure S5: Effective particle size extracted from scattering spectra as a function of size determined by scanning electron microscopy. Dots, data; line, linear fit. The inset shows an example of a diamond particle image and the corresponding largest circle inscribed; the scale bar is $500 \mathrm{~nm}$.

amond particles.

Strictly speaking, to define the eigenmodes of a particle, one needs to perform calculations taking into account its actual shape. From a practical point of view, it may be unnecessary when many various-shaped particles are used. For a single diamond, however, the accuracy of the calculations can be significantly improved by using its actual shape in the model. We illustrate this concept by modeling scattering at an octahedron-like particle, as shown in Figure S6. The octahedron scattering spectrum is in better agreement with the experimental one than the scattering spectrum of a spherical particle. Minor discrepancies that are still present can be caused by deviations of the particle from the ideal octahedron shape. Finally, we applied the effective size determination procedure to the calculated octahedron scattering spectrum. The obtained value of $330 \mathrm{~nm}$ was found to be the same as for the experimental spectrum.

To further investigate the effect of nonsphericity on the scattering resonances, we performed calculations of scattering spectra for a cuboctahedron, a truncated octahedron, an equilateral triangle prism, and a cube. The results of these calculations are shown in Figure S7. We approximated the obtained spectra in the same way as the experimental ones to obtain the effective size values. Depending on the particle shape, the spectra are described in the spherical shape approxi-

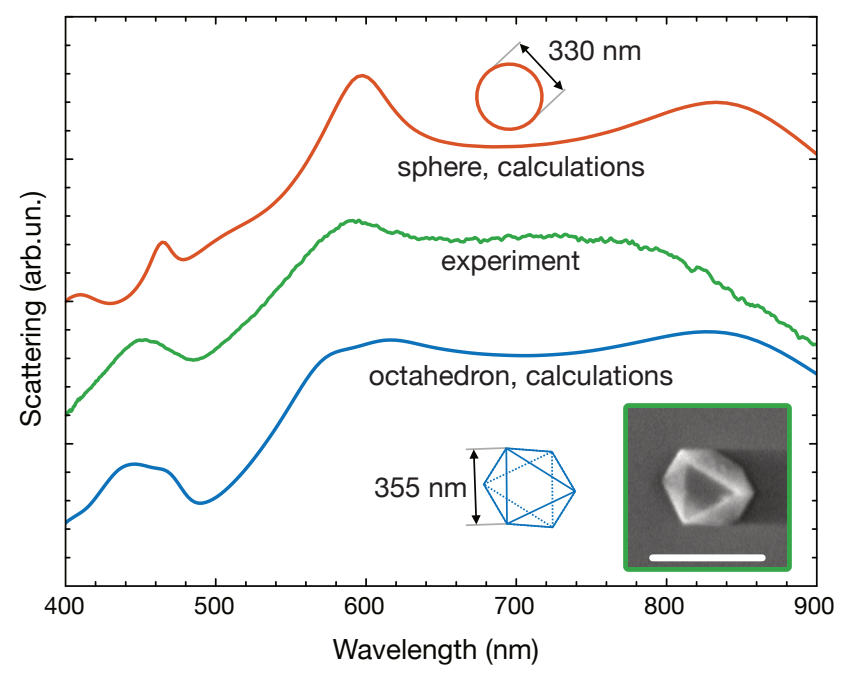

Figure S6: Modeling scattering at an octahedron-like particle. Blue curve, numerically calculated scattering spectrum for a diamond octahedron with an edge of $355 \mathrm{~nm}$. Green curve, experimental scattering spectrum measured for the particle shown in the inset (scale bar is $500 \mathrm{~nm}$ ). Orange curve, numerically calculated scattering spectrum for a 330-nm diamond sphere. All the calculations were performed taking into account the substrate. The spectra are vertically shifted for better perception.

mation to a greater or lesser extent. The shape of an object can be quantitatively characterized by the sphericity parameter [5]:

$$
S=\frac{\pi^{1 / 3}(6 V)^{2 / 3}}{A},
$$

where $V$ is the particle volume and $A$ is the surface area of the particle. Sphericity $S$ takes values from 0 to 1 , and the higher, the closer the particle is to a sphere. The calculated sphericity for each particle shape is shown in the upper right corners in Firure S7. Note that the higher the sphericity of the particle, the better its spectrum is described in the spherical approximation.

\section{Section IV. Lifetime measurements}

The experimental setup for the time-resolved fluorescence measurements is shown in Figure S8. Picosecond pulsed radiation from a diode laser ( $\mathrm{Pi}$ coquant LDH-FA 530XL) is focused on the sample using a plan objective with a focal length of $2 \mathrm{~mm}$ and a numerical aperture of 0.7 . The fluorescence is collected in reflection geometry, separated from the pump using a longpass dichroic mirror 

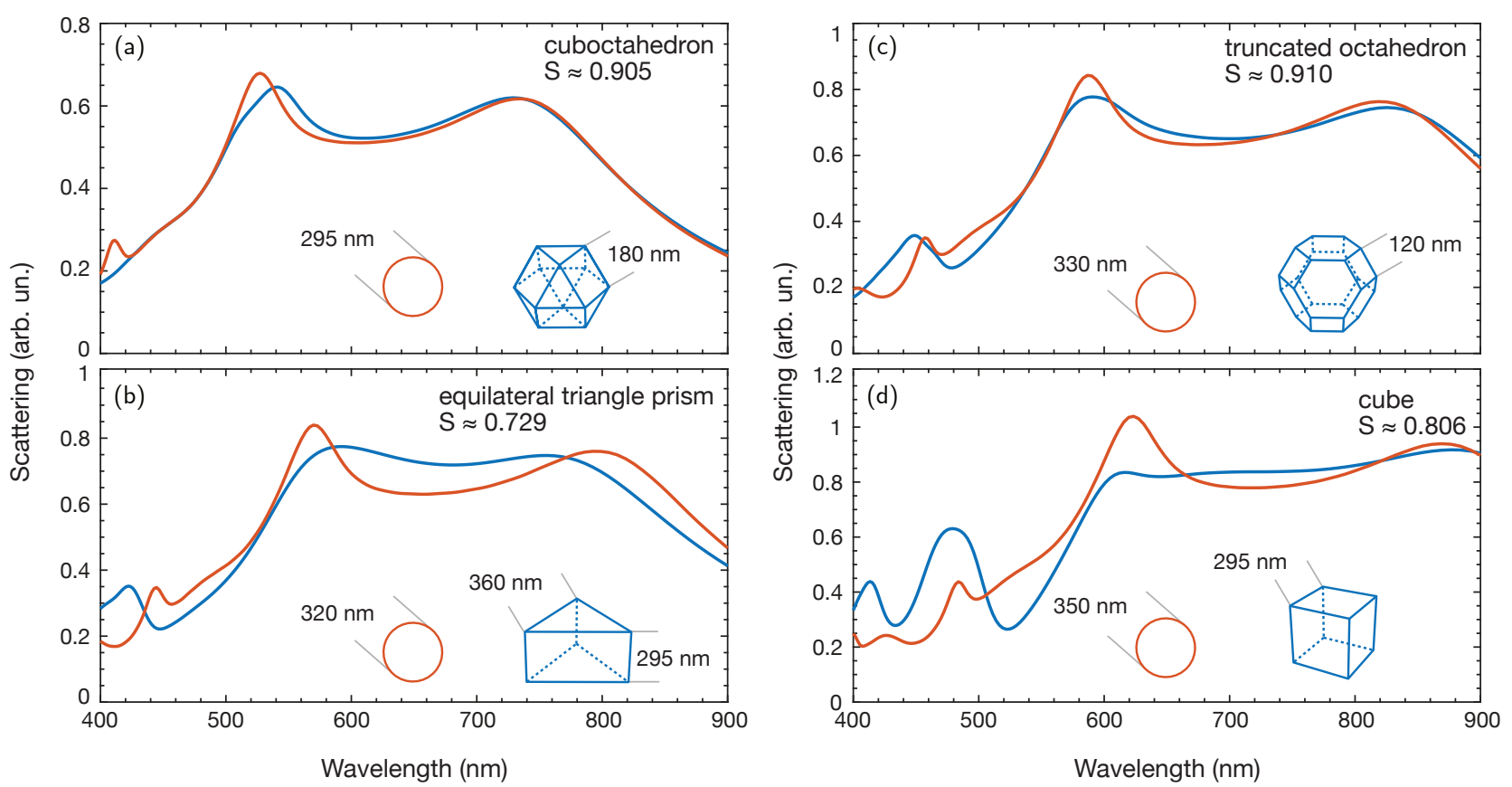

Figure S7: Scattering spectra calculated numerically for (a) cuboctahedron, (b) equilateral triangle prism, (c) truncated octahedron, (d) cube (blue curves) and their approximation by spectra of spherical particles with the particle size and overall intensity used as adjustable parameters (orange curves). The dimensions used in the calculations are shown in the insets; sphericity $S$ is indicated in the upper right corners.

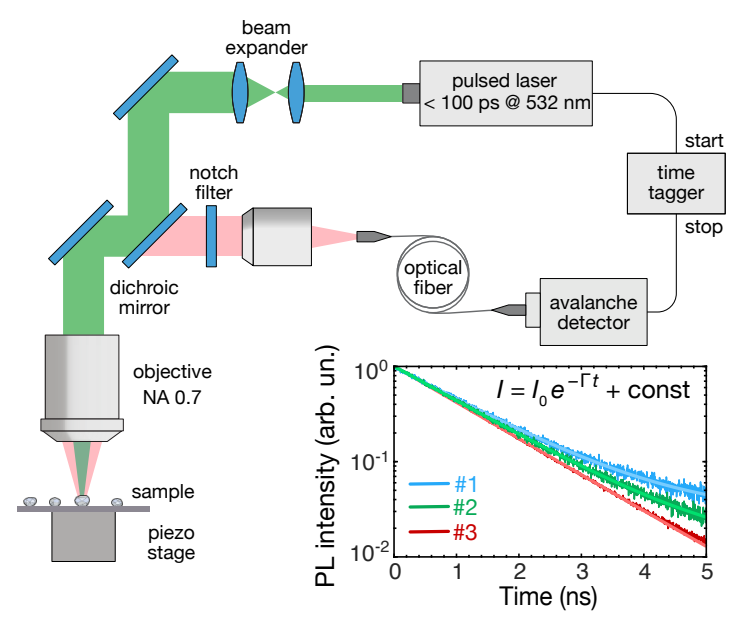

Figure S8: Experimental setup for time-resolved fluorescence measurements. The inset shows the fluorescence time profiles for the particles marked in Figure 2 in the main manuscript.

with a cutoff wavelength of $550 \mathrm{~nm}$ and a notch filter (Semrock NF01-532U-25), and directed into a single photon avalanche detector (MPD PDM PD-100-CTC-FC). Time-correlated single photon counting module (Picoquant Picoharp 300) is used as a time tagger.

The inset of Figure S8 shows the time profiles of the fluorescence signal measured for the parti- cles marked in Figure 2 in the main manuscript. The time profiles were fitted with an exponential decay law $I=I_{0} e^{-\Gamma t}+$ const, where $\Gamma$ is the decay rate and the 'const' term stands for dark counts. Zero time $(t=0)$ corresponds to approximately $1 \mathrm{~ns}$ after the moment of the maximum instrument response. In this way, the rapidly decaying background luminescence signal was removed from the approximation.

\section{Section V. Raman scattering}

The experimental setup used for the Raman scattering measurements is shown in Figure S9a; the same setup was used to obtain the steadystate luminescence spectra. As a source, we use a continuous-wave diode-pumped solid state laser with an output at $532 \mathrm{~nm}$ (Oxxius L1C-532) equipped with an optical isolator and coupled to a single-mode fiber. The fiber output is collimated into a parallel beam with a diameter of $1 \mathrm{~mm}$ and passed through a system of a half-wave plate HWP and a Glan prism GP for power control. A laser line filter (Thorlabs FL532-10) is used to remove remaining optical pump radiation of the laser. A thin glass plate BS is used to direct part of the op- 
(a)

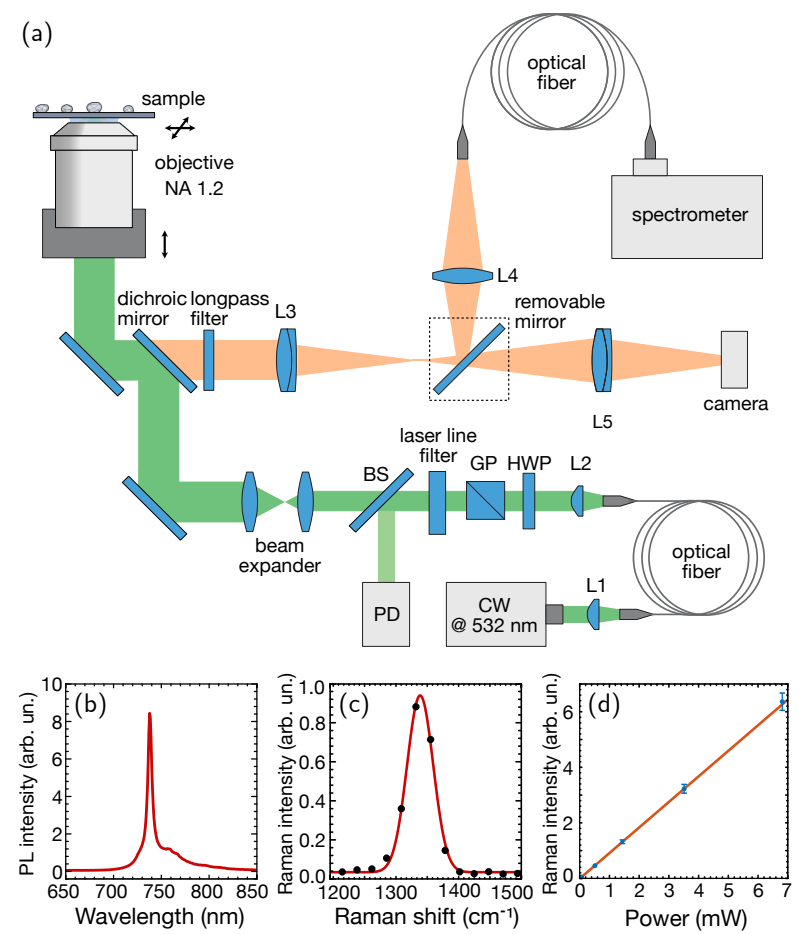

Figure S9: (a) Experimental setup for the steady-state measurements of photoluminescence and Raman scattering. L1, L2, coupler/collimator lenses; HWP, halfwave plate; GP, Glan - Taylor prism; BS, beam splitter; L3 - L5, lenses. (b) Typical SiV color center luminescence spectrum. (c) Typical Raman scattering spectrum. The dots are measured values, the curve is the Gaussian fit. (d) The dependence of the Raman peak intensity on the excitation power (dots) and its linear fit (line). tical power to a photodiode PD. The beam is then expanded and directed into a water-immersion objective lens with an aperture of 1.2. The sample diamonds are located on a translation stage in the focal plane of the objective. The excitation beam is focused into a spot with a diameter of $0.8 \mu \mathrm{m}$. The luminescence and Raman emission are collected using the same objective lens and separated from the pump using a dichroic mirror (Thorlabs DMLP550) and a longpass optical filter (Thorlabs FELH550). Depending on the position of a removable silver mirror, the sample diamonds are imaged either onto a CMOS camera or the facet of an optical fiber connected to an Ocean Optics QEPro spectrometer.

Photoluminescence and Raman scattering spectra measured for one of the studied particles are shown in Figures S9b and S9c. The spectral resolution of the spectrometer used is approximately $50 \mathrm{~cm}^{-1}$, which is an order of magnitude larger than the typical width of the diamond Raman scattering line $\left(3-4 \mathrm{~cm}^{-1}\right)$ resulting in an observed linewidth of about $50 \mathrm{~cm}^{-1}$ for all particles. The dependence of the Raman peak intensity on the excitation power is shown in Figure S9d. This dependence is linear in the experimental power range for all the studied particles, as expected in the case of spontaneous Raman scattering. To obtain the normalized Raman intensity value, for each particle we found the proportionality constant and divided it by the effective diameter cubed.
[1] K. M. Kondrina, O. S. Kudryavtsev, I. I. Vlasov, R. A. Khmelnitskiy, and E. A. Ekimov, Diam. Relat. Mater. 83, 190 (2018).

[2] C. F. Bohren and D. Huffman, Absorption and scattering of light by small particles (Wiley, 1983), ISBN 9780471293408.

[3] H. Chew, Phys. Rev. A 38, 3410 (1988).
[4] J. Schindelin, I. Arganda-Carreras, E. Frise, V. Kaynig, M. Longair, T. Pietzsch, S. Preibisch, C. Rueden, S. Saalfeld, B. Schmid, et al., Nat. Methods 9, 676 (2012).

[5] H. Wadell, J. Geol. 43, 250 (1935). 